\section{AB0102 IMPAIRMENT OF GRANZYME B-PRODUCING REGULATORY B CELLS EXACERBATED RHEUMATOID ARTHRITIS}

L. Xu, F. Hu, X. Liu, L. Zhu, L. Ren, H. Liu, H. Zhu, Y. Su. Department of Rheumatology and Immunology, Peking University People's Hospital, Beijing, China

Background: Rheumatoid arthritis (RA) is a common and complex autoimmune disease characterized by chronic inflammation and cartilage/bone damage involving numerous cells, such as $\mathrm{T}$ cells, $\mathrm{B}$ cells, chondrocytes, fibroblasts ${ }^{[1]}$. B cells had long been well-demonstrated to participant in the development of RA [2]. Except producing specific antibody and inducing $T$ cell activation, impaired immunosuppressive function of $B$ cells further emphasized their roles in RA recently ${ }^{[3]}$.

Objectives: To investigate whether B cells could produce granzyme B and the potential role in the pathogenesis of Rheumatoid arthritis (RA).

Methods: To reveal the expression of granzyme B in B cells, flow cytometry, PCR and Elispot were performed. The role of IL-21 and anti-BCR stimulation on granzyme $B$ expression was assessed by in vitro stimulation assay. $C D 4^{+} T$ cell-B cell co-culture in the presence of granzyme $B$ neutralizing antibody was performed to demonstrate the function of these cells. Then the levels of granzyme $B$ in $B$ cells between RA patients, OA patients as well as HCs were compared. Next,the correlation analysis between granzyme B-producing B cells and clinical features in RA patients was performed. Finally, the frequencies of granzyme B-producing $B$ cells in RA patients before and after therapy were also evaluated using flow cytometry.

Results: B cells could spontaneously produce granzyme B, which could be perpetuated by IL-21 and anti-BCR stimulation. The frequencies of Th1 and Th17 cells were significantly elevated under the condition of granzyme B blockade when granzyme $B$ was neutralized in $C D 4^{+} T$ cell-B cell co-culture. In RA patients, but not $\mathrm{OA}$ patients and $\mathrm{HCs}$, the frequencies of granzyme-B producing Bregs decreased significantly, which was functionally impaired and negatively correlated with disease activity score 28. Moreover, after effective clinical therapy, the frequencies could recover to nomal levels.

Conclusions: B cells could exert the regulatory functions via granzyme B production. Under RA circumstance, these granzyme B-producing Bregs were impaired and contributed to the disease progression.

\section{References:}

[1] Smolen JS, Aletaha D, McInnes IB, Rheumatoid arthritis, Lancet. 2016 Oct 22;388(10055):2023-2038. doi: 10.1016/S0140-6736(16)30173-8.

[2] Nakken B, Munthe LA, Konttinen YT, et al,B-cells and their targeting in rheumatoid arthritis-current concepts and future perspectives. Autoimmun Rev. 2011 Nov;11(1):28-34. doi: 10.1016/j.autrev.2011.06.010.

[3] Daien Cl, Gailhac S, Mura T, Regulatory B10 cells are decreased in patients with rheumatoid arthritis and are inversely correlated with disease activity. Arthritis Rheumatol. 2014 Aug;66(8):2037-46. doi: 10.1002/art.38666.

Acknowledgements: This study was supported by grants from the Natural Science Foundation of China (81671609, 81671604,31470039).

Disclosure of Interest: None declared

DOI: 10.1136/annrheumdis-2017-eular.2269

\section{AB0103 ANTI-INFLAMMATORY AND IMMUNOMODULATORY EFFECTS BY HUMAN UMBILICAL CORD MESENCHYMAL STEM CELL DERIVED MICROVESICLES IN RATS WITH COLLAGEN INDUCED}

M. Tian, K. Xu, L. Zhang, J. Gao. Rheumatology, Shanxi DaYi Hospital, Shanxi Academy of Medical Sciences, Taiyuan, China

Background: Immunologic deranging and persistent inflammation is closely associated with the arising and developing of rheumatoid arthritis (RA). Increasing investigators demonstrated microvesicles (MVs) derived from mesenchymal stem cell (MSC-MVs) might simulate immune regulation and tissue repair of the parental cells. However,the immunotherapeutic potential of MVs in RA remains unknown.

Objectives: We investigated the therapeutic effects of MSC-MVs in RA model collagen induced arthritis $(\mathrm{CIA})$ rats.

Methods: We tested the therapeutic effects of MSC-MVs on CIA rats,levels of $\mathrm{T}$ helper 17(Th17), regulatory $\mathrm{T}$ cell, and cytokines related, as well as specific transcriptional regulation factor Foxp3 and ROR- $\gamma \mathrm{T}$ analysis was performed.

Results: Here, we show that MSC-MVs administration effectively improve arthritic symptoms, inhibit synovial hyperplasia,thus delaying the progression to inflammatory bone destruction, as effective as their original cells, exerting arthralprotective effects. MSC-MVs treating inhibited the proliferation of $\mathrm{T}$ cell,accelerated the apoptosis. MSC-MVs treating reduce proinflammatory cell Th17, cytokines IL-17, while a incline in the level of an-inflammatory cell Treg,ncytokines TGF- $\beta$. In the spllen and ankle joint of CIA rats, MSC-MVs treating increased the expression level of Foxp3 and coinciding with the ROR- $\gamma$ T suppressed, which, the enhanced therapeutic effects correlated with the increase of dosage in a certain range. Conclusions: MSC-MVs showing anti-inflammatory and immunomodulatory activities on CIA rats, suggesting a new and feasible strategy for protection against RA.

References:

[1] E.M. Gravallese, S.R. Goldring, Cellular mechanisms and the role of cytokines in bone erosions in rheumatoid arthritis, Arthritis Rheum. 43 (10) (2000) 2143-2151.

[2] S. Culshaw, I.B. McInnes, F.Y. Liew, What can the periodontal community learn from the pathophysiology of rheumatoid arthritis? J. Clin. Periodontol. 38(Suppl. 11) (2011) 106-113.

[3] Schett, G., D. Elewaut, I.B. McInnes, J.M. Dayer, and M.F. Neurath, How cytokine networks fuel inflammation: Toward a cytokine-based disease taxonomy. Nat Med, 2013. 19(7): p. 822-4.

Disclosure of Interest: None declared

DOI: 10.1136/annrheumdis-2017-eular.3462

\section{AB0104 ALTERATIONS OF SPLICEOSOME COMPONENTS IN LEUKOCYTES FROM PATIENTS WITH RHEUMATOID ARTHRITIS INFLUENCE THEIR AUTOIMMUNE AND INFLAMMATORY PROFILE, AND THE DEVELOPMENT OF CARDIOVASCULAR DISEASE}

P. Ruiz-Limon ${ }^{1}$, C. Perez-Sanchez ${ }^{1}$, R. Ortega-Castro ${ }^{1}$, S. Pedraza-Arevalo ${ }^{2}$, M. del Rio-Moreno ${ }^{2}$, Y. Jimenez-Gomez ${ }^{1}$, I. Arias de la Rosa ${ }^{1}$,

M. Ábalos-Aguilera ${ }^{1}$, M. Aguirre ${ }^{1}$, P. Segui ${ }^{1}$, E. Collantes ${ }^{1}$, A. Escudero ${ }^{1}$

J. Castaño ${ }^{2}$, R.M. Luque ${ }^{2}$, N. Barbarroja ${ }^{1}$, C. Lopez-Pedrera ${ }^{1} .{ }^{1}$ Rheumatology

Service; ${ }^{2}$ Department of Cell Biology, Physiology, and Immunology, IMIBIC/Reina Sofia Hospital/University of Cordoba, Cordoba, Spain

Background: A significant percentage of genetic and inflammatory diseases derive from splicing alterations. Therefore, the understanding of what modifications in spliceosome determine an alternative splicing and its association with the development of such pathologies is of critical importance.

Objectives: To identify the alterations present in the spliceosome machinery of patients with rheumatoid arthritis (RA),its influence on the development and activity of the disease and their atherothrombotic profile.

Methods: An array of selected components of the major- $(n=12)$ and minorspliceosome $(n=4)$ and associated splicing factors $(n=28)$ was developed, and their expression levels were evaluated using a Fluidigm methodology, in purified leukocytes from 14 RA patients and 14 healthy donors (HD). In parallel, an extensive clinical/serological evaluation was performed. Carotid intimate media thickness (CIMT) was used as atherosclerosis marker. Endothelial activity was monitored by laser-doppler flowmetry, and pro-inflammatory and oxidative stress markers were quantified. Association of these splicing components with clinical and analytical features were investigated.

Results: A significant alteration in various components of spliceosome and splicing factors was found in all the leukocytes subtypes from RA patients vs HD. Interestingly, we found a specific altered profile of splicing factors and spliceosome components when compared monocytes (CA150, PRP8, SRM160, U2AF1, RNU4atac, PTBB1, RAVER1, RBM17, SRSF4, SRSF10), lymphocytes (RNU12, RNU4, RNU6, PRP8, MAGOH, NOVA1, SRSF3) and neutrophils (RNU11, RNU6, SC35, RBM3).

Altered levels of various spliceosome elements in monocytes were associated with the presence of atheromatous plaques, while in neutrophils were found related to radiological involvement. In lymphocytes, the alteration of these components were linked to the positivity for Rheumatoid Factor and anti-CCPs antibodies, indicating that modifications in the spliceosome machinery could contribute to the increase in the production and assembly of autoantigens, inducing autoantibody production.

Correlation studies showed a significant relationship between altered levels of various spliceosome components in different leukocyte subtypes and high disease activity (DAS, HAQ), increased expression of proinflammatory mediators (CRF, TF, TNF, IL-8, TLR4) and oxidative stress markers (peroxides, GPX, SOD) as well as with parameters associated with insulin resistance.

Conclusions: These results reveal that there is a significant alteration of spliceosome components in RA patients that could be associated with the development and activity of this autoimmune condition, and influence mechanisms that drive the development of cardiovascular disease. Studies in progress will help to clarify the physiological implications of these findings, which could constitute new diagnostic biomarkers, as well as new therapeutic tools for the treatment of RA.

Acknowledgements: Funded by CTS7940, and ISCIII (PI15/01333, CP15/00158, and RIER RD16/0012/0015).

Disclosure of Interest: None declared

DOI: 10.1136/annrheumdis-2017-eular.4907

\section{AB0105 DOXYCYCLINE AND DEXAMETHASONE-INDUCED REPROGRAMMING OF PERIPHERAL BLOOD MONONUCLEAR CELLS IN A MODEL OF ARTHRITIS WITH THE SYSTEMIC MANIFESTATIONS IN WISTAR RATS}

P. Litvitskiy ${ }^{1}$, V. Sakharov ${ }^{1}$, E. Alexeeva ${ }^{2}$, N. Mayansky ${ }^{2}$, R. Zakirov ${ }^{2}$ ${ }^{1}$ Pathophysiology, Sechenov First Moscow State Medical University; ${ }^{2}$ Scientific Center of Children's Health, Moscow, Russian Federation

Background: The systemic juvenile idiopathic arthritis (SJIA) is a problem with high social significance all over the world. Targeted cell reprogramming becomes one of the most important lines for modern medicine and can be regarded as 